\title{
Using a Microcontroller for Sonar Ranging
}

\author{
Hesham Shaalan, Ph.D., and Darrell Morris \\ School of Technology \\ Georgia Southern University \\ Statesboro, Georgia
}

\section{Introduction}

The Senior Project is the capstone course in the Electrical Engineering Technology (EET) curriculum at Georgia Southern University. It consists of an individually chosen project that is taken from concept through design to a finished state. It includes related CAD, specifications, theory of operation, and oral/written reporting ${ }^{1}$.

The purpose of the Senior Project described in this paper is to design a system that will indicate to a driver how close an object is to the back of the vehicle. The system design is based on using a microcontroller and an ultrasonic ranging unit mounted on the back of the vehicle. The distance to an object can be calculated based on the time it takes for a transmitted pulse to bounce off of the object and return to the transducer. This distance can then be displayed to the driver by either audio or visual means. Figure 1 shows a block diagram of the overall system.

The microcontroller is a device similar to the microprocessor, but it is used in control oriented applications. It is a single integrated circuit that includes a CPU, RAM, ROM, timers, and interface circuitry. Microcontrollers can be found in products such as automobiles, home appliances, and industrial equipment ${ }^{2}$.

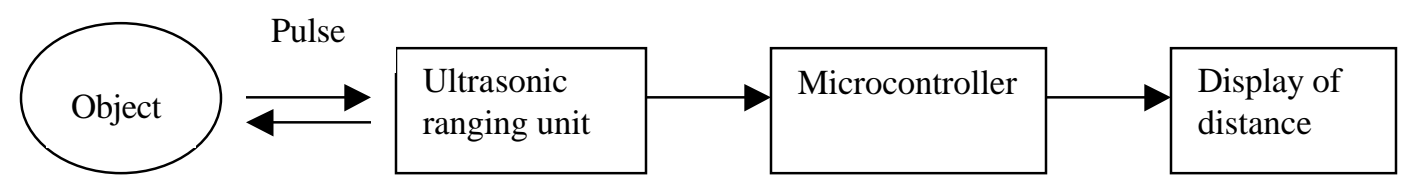

Figure 1: block diagram of the overall system

2. System Design

The following design criteria was used in selecting a microcontroller for this project:

1. It must be capable of being reprogrammed easily to facilitate testing and program debugging.

2. It must have at least one 16-bit timer.

3. It must have at least 9 lines available for output and one available for input (10 total I/O lines). 
4. It must be based on the Intel 8051 architecture to facilitate programming in a familiar language.

5. It must be cost effective.

In 1981, Intel Corporation introduced an 8-bit microcontroller called the 8051. The microcontroller had 128 bytes of RAM, 4K bytes of on-chip ROM, two timers, one serial port, and four ports all on a single chip. The 8051 became widely popular after Intel allowed other manufacturers to make and market other versions of the 8051 as long as they remain codecompatible. This led to many versions of the 8051 with different speeds and amounts of on-chip ROM marketed by several manufacturers such as Atmel.

The microcontroller used for this project is the Atmel A T896C2051 (hereafter referred to as the 2051). This particular microcontroller was chosen for its flash memory capability over EEPROM microcontrollers, which require a more expensive burner. The 2051 is a 8051-based 8bit processor with $2 \mathrm{k}$ bytes of program memory, $15 \mathrm{I} / \mathrm{O}$ lines, and two 16 bit timers, all of which fit well with the listed criteria ${ }^{3}$. In addition, the cost of the microcontroller was only $\$ 4.00$. The program editor was obtained as a trial version downloaded from the Internet. Although several flash programmer designs are available for free download, the programmer was purchased to ensure that it would function without problems.

In choosing a transducer, range was the only consideration. There are a limited number of range-sensing transducers, some of which are based on infrared and some on ultrasonic detection. The infrared range detectors that were examined had a range limited to 3 or 4 feet. However, the ultrasonic detectors were found to have a suitable range up to 35 feet in some cases. The detector chosen for the project is the Polaroid 600 Series Instrument Grade Transducer driven by the Polaroid 6500 Series Sonar Ranging Module ${ }^{4}$.

The Polaroid equipment has been in use for several years in Polaroid Instamatic cameras for the purpose of auto focus, thus the design has proven to be reliable. The advertised range of the Polaroid ranging module and transducer is 35 feet, with the ability to distinguish between objects 3 inches apart. Since the display will show increments of 1 foot up to 8 feet, this accuracy and range is more than adequate.

Since the transducer requires $200 \mathrm{~V}$ at all times and $2000 \mathrm{~mA}$ at $400 \mathrm{~V}$ for the pulse, it was decided that in the interest of saving time, it would be more advantageous to use the Polaroid Ranging Module, rather than design one. The ranging module require s one input, INIT, to initiate a pulse train of 16 pulses at a frequency of $49.4 \mathrm{kHz}$. Upon receiving the first pulse back to the transducer, the module sends a signal, ECHO, to the controlling device.

To provide feedback for distance, either an audio signal from a speaker or a visual display could be used. A visual display was chosen in order to prevent the feedback from being confused with other noises which may be in the background, such as music from the vehicle's radio, or noise from outside the vehicle. The next choice was whether to use a numerical display, such as a seven-segment display, or use a meter-type display, such as a bar graph. The bar graph was chosen so that it could be placed in the rear window of the vehicle, allowing the driver to see 
it while looking in the rear view mirror or through the back glass and get the same information on distance.

\section{Microcontroller Program}

Assembly language is a computer language lying between the extremes of machine language and high-level language ${ }^{2}$. Typical high-level languages like $\mathrm{C}++$ use words and statements. Machine language is a series of binary codes representing computer instructions. Assembly language replaces the binary codes of machine language with easy to remember "mnemonics" that facilitate programming.

The program for the microcontroller is the core of the system. The beginning of the program contains the initialization statements for timer mode, timer control, and program status word. The I/O ports are also initialized to ensure their state at start up. Since the microcontroller is capable of sinking greater current on the I/O pins than it can source, l's are used to initialize the ports and turn off all LED's. The control then jumps to the main program where the ECHO input pin is cleared for reception and the INIT pin is set low to send the command to the ranging module to pulse.

The timer is started and control is passed to the timer subroutine. In the timer subroutine, the timer runs until it has timed out twice, even if a return pulse is detected. This provides a steady rate of 7 pulses per second for detection. When a return pulse is detected, the ECHO pin goes high and control is passed to the display subroutine while the timer remains running.

In the display subroutine, a status flag is set to indicate that the ECHO has been received. The high byte of the 16-bit timer is captured and placed into the accumulator for comparison. This value is compared with numbers that correspond to roughly one-foot increments up to ten feet, and the appropriate bits are set in a register. After comparison to all ten increments, the number in the register is moved to the accumulator where it is complemented (to account for sinking outputs) and written to the output ports that turn on the LED's. Control is passed back to the timer subroutine to count the timer overflows until two have been reached.

Once the timer has overflowed twice, control returns to the main program where the timer is stopped, the ECHO status flag is cleared, and the timer high and low bytes are cleared. The program then begins a new cycle, clearing the ECHO pin and sending a pulse out on the INIT pin.

\section{Conclusion}

When backing up in a vehicle, it is not always possible to tell how close an object is to the back bumper. This paper presented a Senior Design project for a system that indicates to a driver how close an object is to the back of the vehicle. The distance to an object was calculated by using a microcontroller and an ultrasonic ranging unit mounted on the back of the vehicle. This distance can then be displayed visually to the driver. 
This project enabled the student to use the knowledge acquired while in the EET program to perform a project from start to finish. Some of the engineering skills used by the student include testing and troubleshooting. This should enable the student to work on projects in the workplace as an individual or as a team member.

\section{References:}

1. Georgia Southern University Undergraduate \& Graduate Catalog, 2001-2002 edition.

2. The 8051 Microcontroller, 3rd Edition, by I. Scott MacKenzie, Prentice Hall, 1999.

3. Atmel AT896C2051 Microcontroller User Guide, Atmel Corporation.

4. Polaroid 6500 Series Sonar Ranging Module Specifications, Polaroid Corporation.

Biographies:

HESHAM SHAALAN, Ph.D., is an Assistant Professor of Electrical Engineering Technology at Georgia Southern University, Statesboro, Georgia. He received his BSEE and MEE from the University of Houston in 1986 and 1987, respectively, and his PhD from Virginia Tech in 1992. He is a member of Eta Kappa Nu Honor Society, American Society of Engineering Education (ASEE), and Senior member of the Institute of Electrical and Electronics Engineers (IEEE).

DARRELL MORRIS recently graduated from Georgia Southern University with a Bachelor's degree in Electrical Engineering Technology. He is currently employed with Briggs \& Stratton. 